\title{
EDITORIAL ARTICLES ROADEF 2007
}

This issue contains a collection of six selected articles that have been prepared by participants of the joint Conference FRANCORO V/ROADEF 2007. This event took place in Grenoble from February 20 to 23, 2007, and brought together almost 400 researchers who presented 237 papers, addressing most of the existing topics in Operations Research and Decision Support.

The given collection of papers is presented in two groups, consisting of three articles each. The first group concerns the challenge ROADEF 2007.

The industrial challenge of the French OR society (challenge . roadef .org) was dedicated in 2007 to a subject proposed by France Telecom on "Technicians and Interventions Scheduling for Telecommunications". This subject was co-sponsored by ILOG and EURODECISION. 31 teams from 15 countries worldwide had competed for the qualification stage in 2 categories (13 Juniors and 18 Seniors) from July 2006 to January 2007. 17 teams were selected and ranked to compete in the final stage. 9 grand finalists (4 Juniors and 5 Seniors) were invited to the joint conference to present their work. Three papers from this group have finally been selected for this special issue.

S. Pokutta and G. Stauffer present an original method using a ResourceConstrained Project Scheduling Problem (RCPSP) formulation of the workforce scheduling problem. They use a metaheuristic strategy based on an activity list representation and associated schedule generation schemes. Interventions are placed using a perfect matching formulation with additional constraints, solved by branch-and-cut.

S. Boussier, H. Hashimoto, M. Vasquez and C. Wilbaut present a Greedy Randomized Adaptive Search Procedure (GRASP) with dynamic update of intervention selection criteria. Several lower bounds (two-dimensional level packing lower bound, linear programming...) are used to assess the solution quality.

C.A.J. Hurkens proposes a Mixed Integer Programming (MIP) method to obtain outstanding results. In a preprocessing phase the number of technicians required in each team is estimated through a first MIP. Then a lower bound is computed via a second MIP by allowing job preemption. Multiple solutions are constructed by iteratively solving a third Integer Program (matching formulation) with different parameter values but without introducing randomness.

These three teams are ranked in the all-category standing as 5th (Pokutta and Stauffer), 4th (Boussier, Hashimoto, Vasquez and Wilbaut) and 1st (Hurkens). The 4 th is also the 1 st in the Junior category. 
The second group of papers is dealing with three rather different subject areas. N. Dridi, H. Hadda et S. Hajri-Gabouj consider a hybrid flowshop scheduling problem with dedicated machines. The objective is to minimize the makespan. They present two polynomial sub-problems and propose a heuristic approach to solve the general problem. Numerical experiments show the quality of the approach compared with proposed lower bounds.

Y. Caseau develops in his article a simulation method, called GTES, with the aim to analyze the dynamics of general agent-based systems. The approach is based on three different techniques: Monte-Carlo methods, game theory and local search methods. In particular, genetic algorithms are used to enhance the search for the Nash equilibrium in an environment of non-cooperative games. Illustrative examples are included that come from the telephone market.

E. Duchêne, S. Gravier and J. Moncel present new results on a combinatorial game, called "Solitaire Clobber". This is a one-player game, which is played on a graph. Starting with some initial configuration of "stones" on the vertices, one would like to find a sequence of "moves" so that one is left with the minimum number of stones on the graph at the end of the game. In this article, the type of graph is extended to complete multi-partite graphs.

Christian Artigues

Van-Dat Cung

Gerd Finke

Bernard Penz 